\title{
DÜBLIN
}

Technological University Dublin

ARROW@TU Dublin

2005-01-01

\section{Electroabsorption Studies of Structurally Modified Fullerene Thin Films}

\author{
Gerald Farrell \\ Technological University Dublin, gerald.farrell@tudublin.ie \\ Gordon Chambers \\ Technological University Dublin, Gordon.chambers@tudublin.ie \\ Hugh Byrne \\ Technological University Dublin, hugh.byrne@tudublin.ie
}

Follow this and additional works at: https://arrow.tudublin.ie/radcon

Part of the Physics Commons

\section{Recommended Citation}

Farrell, G., Chambers, G. \& Byrne, H. (2005) Electroabsorption studies of structurally modified fullerene thin films. Proceedings of Opto Ireland 2005. SPIE vol. 5826, pg. 232. doi:10.1016/j.jlumin.2004.09.106

This Conference Paper is brought to you for free and open access by the Radiation and Environmental Science Centre at ARROW@TU Dublin. It has been accepted for inclusion in Conference papers by an authorized administrator of ARROW@TU Dublin. For more information, please contact arrow.admin@tudublin.ie, aisling.coyne@tudublin.ie, gerard.connolly@tudublin.ie.

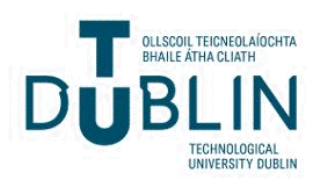




\title{
Electroabsorption studies of structurally modified fullerene thin films
}

\author{
G. F. Farrell ${ }^{\mathrm{a} *}$, G. Chambers ${ }^{\mathrm{b}}$, H.J. Byrne ${ }^{\mathrm{a}}$ \\ ${ }^{a}$ Focas Institute, Dublin Institute of Technology, Kevin Street, Dublin 8, Ireland \\ ${ }^{\mathrm{b}}$ School of Physics, Dublin Institute of Technology, Kevin Street, Dublin 8, Ireland
}

\begin{abstract}
Ultraviolet (UV/Vis) and Electroabsorption (EA) spectroscopy is used to examine and differentiate between intermolecular and intra molecular excited state species in fullerene films. Charge Transfer (CT) states are identified at $2.4 \mathrm{eV}$ and $2.7 \mathrm{eV}$ and dipole moments are calculated. Thermal annealing of $\mathrm{C}_{60}$ films is monitored in situ using absorption spectroscopy and electroabsorption spectroscopy. Recorded spectra display both some temperature dependent and partially irreversible effects, indicating the occurrence of an annealing process. EA shows that the CT states associated with the transferring an electron from the HOMO of one molecule located at the $(0,0,0)$ position to the LUMO of its nearest neighbour in the $(1 / 2,1 / 2,0)$ have been modified as a result of the annealing process. Confirmation of this structural change due to the annealing process is provided by previously reported X-Ray crystallographic work.
\end{abstract}

Keywords: $\quad$ Fullerenes, Electroabsorption, Lattice Structure, Annealing

\section{INTRODUCTION}

Since the discovery in 1985 of the Buckminster fullerene by Kroto et al [1] a great deal of research has been afforded to the area of fullerene research. In fact with the advent of large scale production methods, the first commercial applications of fullerenes have started to appear. Indeed the Frontier Carbon Corporation (FCC) with the backing of commercial giants, such as the Mitsubishi Corporation, have now applied fullerene technology to a number of sporting goods, including bowling balls and golf clubs. With the utilisation of new combustion methods it is estimated that by the year 2007, FCC will be producing 1,500 tonnes of fullerene per annum. This will greatly reduce production costs and consequently result in an increase in potential applications. However these sporting applications of $\mathrm{C}_{60}$ are not those which were anticipated, i.e. ones based on their electronic properties. The potential of these future electronic applications require a complete understanding of the material itself. Being able to measure and control the unique properties of this material is of utmost importance.

The first EA analysis of $\mathrm{C}_{60}$ was preformed by Pichler et al in 1991[2]. Since then numerous studies have been preformed and it is generally accepted that the EA spectrum of the solid state of $\mathrm{C}_{60}$ is dominated by charge transfer states [3]. It has also been observed that these features become enhanced at temperatures below the well-known orientation phase transition temperature of solid state $\mathrm{C}_{60}$ at $249 \mathrm{~K}$ suggesting that changes in the orientational order due to the phase transition can modify or control the relative contribution of inter- and intra- molecular interactions. Indeed several methods in the past have shown that the structural and electronic changes incurred due to the phase transition can be shifted to higher temperature [4][5]. An example is the formation of clathrates in which $\mathrm{C}_{60}$ crystals are exposed to various neutral solvents, which intercalate themselves into the fcc lattice arrangement of $\mathrm{C}_{60}$, thereby reducing the symmetry of the crystals to an orthorhombic phase. The overall net effect of this reduction in symmetry is to shift the absorption edge to higher energies and the orientational phase transition at $249 \mathrm{~K}$ to a higher temperature dependent on the degree of intercalation and the intercalating molecule itself.

In addition to solvent inclusion it has been shown that the lattice structure of $\mathrm{C}_{60}$ can be affected by thermally annealing films. By annealing $\mathrm{C}_{60}$ thin films a partial phase transition to a hcp crystalline phase occurs [6]. This transition results in a decrease in the optical oscillator strengths, which is also coupled by a red shift of up to $50 \mathrm{~nm}$ in the absorption spectrum. A number of studies on annealed films have been reported [6,7] where it was seen that UV/Vis 
and IR spectra show no indication of any chemical alteration of the sample but rather an apparent structural change is observed [7], similar to that reported for $\mathrm{C}_{60}$ clathrates. [8]

This work will investigate the effects of the structural modification of $\mathrm{C}_{60}$ thin films primarily by changing the crystallinity of fullerene thin films via a systematic annealing procedure. Characterization of these films will primarily involve using UV/Vis and electro - absorption (EA) measurements. In both cases the aim is to correlate changes in charge transfer states to the reported structural changes in the $\mathrm{C}_{60}$ lattice.

\section{METHODOLOGY}

Thin films of $\mathrm{C}_{60}$, (99.98\% pure as purchased from SES Research) $240 \mathrm{~nm}$ thick, were produced by vacuum deposition onto $12-\mathrm{mm}^{2}$ Indium-Tin oxide (ITO) covered glass substrates. To ensure that the films were not annealed during fabrication, the distance between the substrate and the evaporation boat was maintained at $10 \mathrm{~cm}$. A transparent aluminium top electrode ( $15 \mathrm{~nm}$ thick) was then deposited through a shadow mask, onto the surface of the $\mathrm{C}_{60}$ film. This afforded good electrical contact with the film while being transparent enough to enable UV/Vis and electroabsorption measurements

Absorption spectra were recorded using a Perkin-Elmer Lambda 900 UV/Vis/NIR incorporating a Linkam Scientific THS heating stage. This stage has an operational temperature range from $-198{ }^{\circ} \mathrm{C}$ to $600{ }^{\circ} \mathrm{C}$. A selection of the films where annealed by post-deposition heating in the heating stage at various temperatures under a nitrogen atmosphere. The maximum annealing temperature of $175 \mathrm{C}$ was chosen so as to be sufficiently below the sublimation temperature.

The electroabsorption (EA) measurements were preformed using a homemade system, similar to that described by Boxer et al.[9] The EA spectrometer consisted of a Lot-Oriel 350W Xenon arc lamp focused onto the entrance slit of a CVI 1/4-M Monochromator, with a 1200 lines $/ \mathrm{mm}$ grating. The resulting emergent light was then passed through a polariser and focused onto the sample located inside a Linkam Scientific THS cooling/ heating stage. The transmitted light was then measured using a Hamamatsu 1P28A photomultiplier tube, which was in-turn connected to an EG\&G 5210 lockin Amplifier tuned to the second harmonic of the applied electric field. The electric field, (typically 500KV), was generated by a SRS DS345 signal generator. A LabVIEW program was used to control the instrumentation and also for data accumulation. The electroabsorption spectra were obtained by measuring the change in transmission $(\Delta \mathrm{T})$ under the influence on an electric field, $(2 w, w=190 \mathrm{~Hz})$ which was normalised to the transmission $(\mathrm{T})$ of the same sample. The following relationship $\Delta \mathrm{T} / \mathrm{T}=-d \Delta \alpha$ was used, where $d$ is the film thickness and $\alpha$ is the absorption coefficient.

\section{RESULTS AND DISCUSSION}

Figure. 1 (a) shows the absorption spectrum of a thin film of $\mathrm{C}_{60}$. Evident from this spectrum are a number of features, which are molecular in origin and some which are the result of the solid state environment. In particular the lowest energy transition in the solid state, which has maximum at $\sim 2.0 \mathrm{eV}$, possesses a vibrational structure similar to that of the isolated molecule [10]. Furthermore, all of the higher order, allowed molecular transitions appear in the solid state spectrum, although slightly red shifted. On this basis, the solid state of $\mathrm{C}_{60}$, appears to behave like a classical molecular solid in which the molecular wavefunctions are minimally perturbed, the principal influence of the crystalline environment being in symmetry breaking. However, there is a broad, relatively strong absorption centred around 2.75 $\mathrm{eV}$ which is absent in solution and gas phase EELS spectra $[11,12]$ hence suggesting that it is not molecular in origin but rather the result of the solid state environment. Further to this photoconductivity and electro-absorption similarly indicate a photoexcited species which is not molecular in origin $[13,14]$. Kazaoui et al [13], have demonstrated, via electro-absorption that the feature at $\sim 2.75 \mathrm{eV}$ derives from a series of charge transfer states, whereby the transition occurs between the HOMO of one molecule to a higher unoccupied state whose wavefunction derives from a mixture of the molecular state with those of its next nearest neighbours. It is thus now widely accepted [15] that this feature consists of two distinct absorptions, one at $\sim 2.43 \mathrm{eV}$ and the other at $\sim 2.75 \mathrm{eV}$. These are due to the solid state's influence on the electronic properties of the molecule, hence it is not molecular in character but instead is the result of an intermolecular charge transfer excited state. 


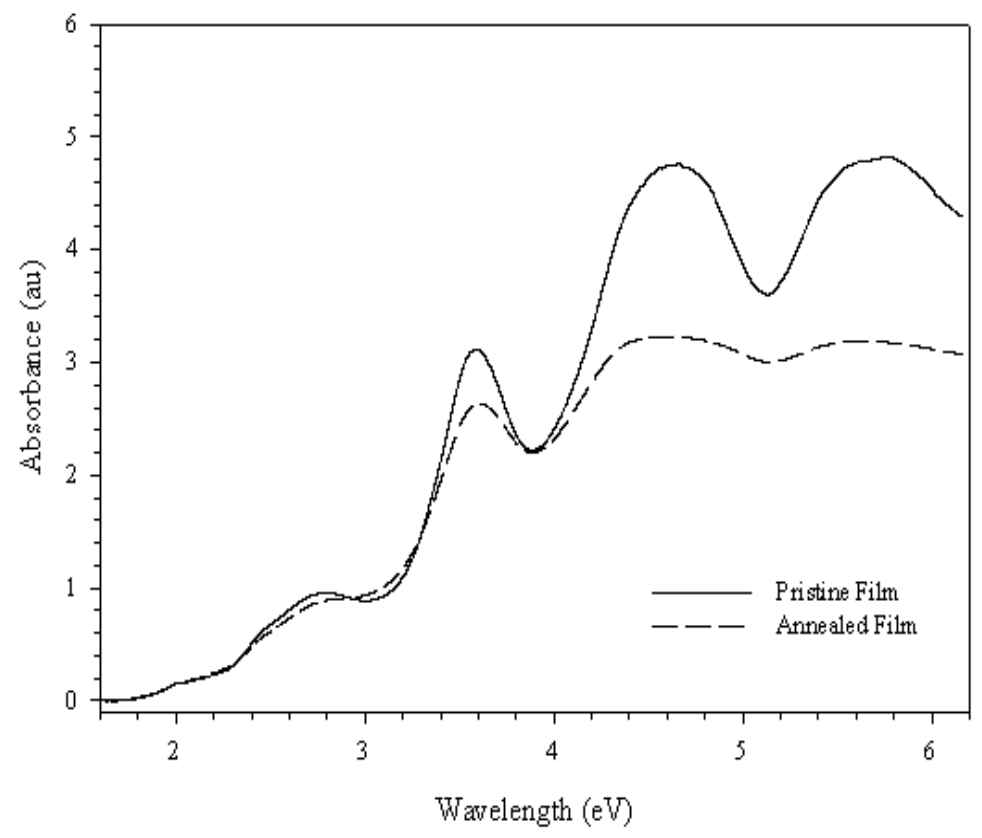

Fig.1 (a) Shows the absorption spectrum of a pristine $\mathrm{C}_{60}$ film (b) Shows the affect of annealing a $\mathrm{C}_{60}$ film for 24 hours (dotted line)

As previously mentioned it has been shown as early as 1994, that the absorption spectrum of solid state $\mathrm{C}_{60}$ can be altered due to external structural modification of the films [6]. Initially such work focused upon the thermal annealing of $\mathrm{C}_{60}$ thin films in which it was reported that a marked decrease in the oscillation strengths of transitions was observed due to the annealing process [11].

Figure 1(b) shows the absorption spectrum obtained from a film which was annealed for 24hours and indeed there is an overall decrease in the oscillation strengths of the features between $3.1 \mathrm{eV}$ and $6.2 \mathrm{eV}$. Although less noticeable there is also a decrease in intensity of the features at $2.43 \mathrm{eV}$ and $2.75 \mathrm{eV}$. This decrease suggests a possible reduction in the charge transfer processes associated with these transitions. There is also a continuous red shift of these solid-state features (although not entirely evident from figure 1). This apparent shifting accompanied by the loss in intensity can be attributed to a closer packing of the molecules in the solid state phase. A theoretical analysis of the effects of crystal packing on both the spectral positioning and oscillator strengths of the $\pi-\pi^{*}$ transitions of $\mathrm{C}_{60}$ in the solid state has been performed by $\mathrm{Wu}$ and Ulloa [16]. The analysis indicated that the oscillator strength and spectral positioning of transitions can have considerable anisotropy due to different Coulomb interactions between molecules in different packing arrangements. This predicted sensitivity of the optical absorption spectrum to crystal packing is consistent with the variations observed in these thermally annealed films

Although these effects, in particular the reduction in oscillator strength is pronounced in the films prepared for optical absorption it was unknown whether or not these reductions would be observable in films which were prepared for the electroabsorption measurements. Of primary concern was whether or not the top transparent Al electrode would have an adverse affect on the flexibility of the lattice structure of the $\mathrm{C}_{60}$ film. To avoid any discrepancies both sets of films were annealed under the same conditions. Another factor which needs to be considered in comparing EA with $\mathrm{UV} / \mathrm{vis}$ is the wavelength range of the electroabsorption spectrophotometer. Since optical glass is utilised in its construction many of the drastic changes observed are currently outside its working range, in particular the 3.2 to $6.2 \mathrm{eV}$ region. However as already mentioned above there are large observable changes in the oscillation strengths at $2.43 \mathrm{eV}$ and $2.75 \mathrm{eV}$. Consequently this work will concentrate on this region, since these features which have been attributed to solid state, relate to the degree of communication between adjacent molecules. Figure 2 shows that the same decrease in absorption oscillation strengths as observed for the reference films in figure 1 is seen for the films which where prepared for the electroabsorption measurements. 


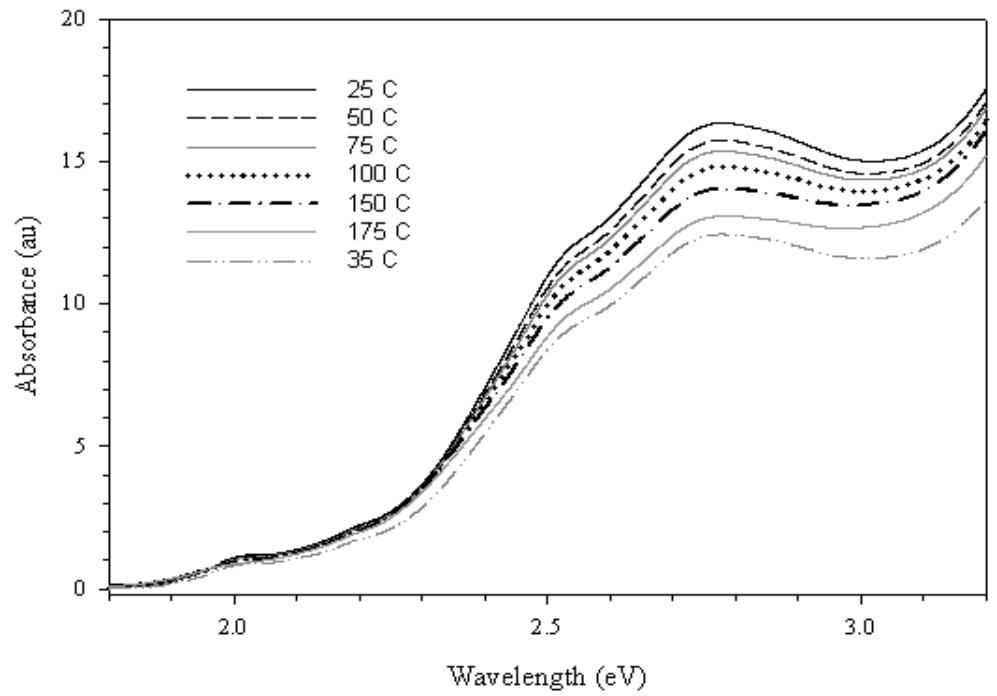

Figure 2 Shows the affect of annealing on samples heated to various temperatures. As can be readily observed there is a gradual decrease in oscillation strength with increasing temperature

Figure 2 shows the absorption spectra of a $\mathrm{C}_{60}$ thin film at different temperatures. It is observable that there is a gradual decrease in the oscillation strengths with increasing temperature. Although part of this reduction in oscillation strength is a temperature dependent effect of the individual absorption bands, closer inspection shows that the absorption spectra of the $\mathrm{C}_{60}$ film at room temperature after annealing shows a significant reduction in strength in comparison to the absorption spectra of the pristine film. By fitting a model of summated Gaussian band- shapes to the above data as show in figure 3 it is possible to monitor the changes of individual electronic transitions.

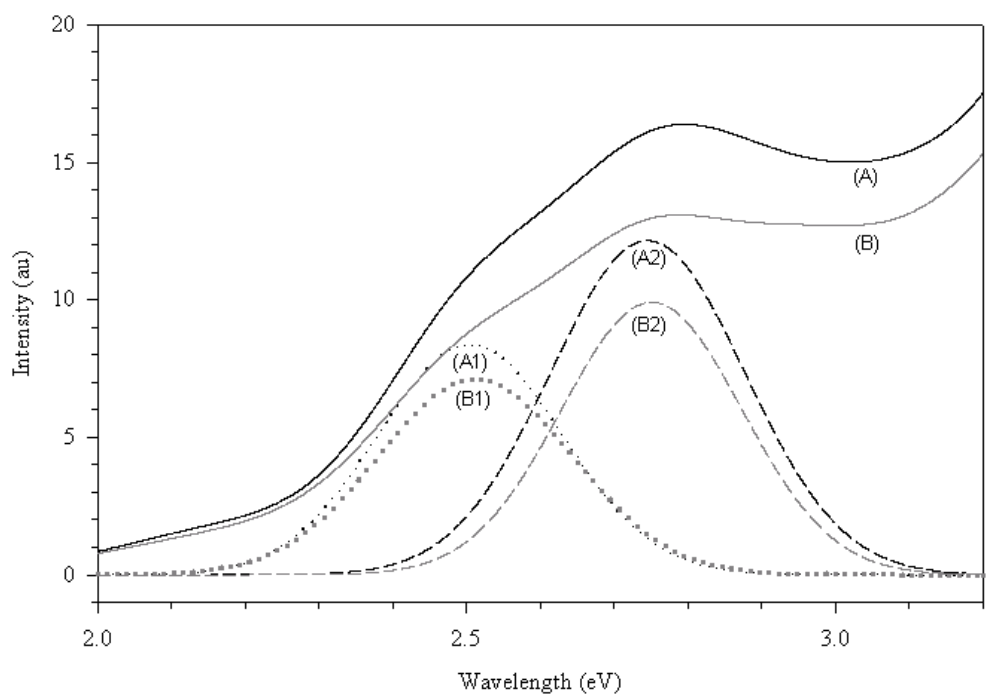

Figure 3 Shows the absorption spectrum of the pristine $\mathrm{C}_{60}$ at $25^{\circ} \mathrm{C}$, curve $\mathrm{A}$, and at $175^{\circ} \mathrm{C}$, curve B. Also show are two Gaussian bands (fits to the spectra) centred at $2.51 \mathrm{eV}$ and $2.75 \mathrm{eV}$.

Figure 3 shows the absorption spectrum of a $\mathrm{C}_{60}$ film at $25{ }^{\circ} \mathrm{C}$, shown as curve $\mathrm{A}$, and also at $175^{\circ} \mathrm{C}$, shown as curve B. Associated with each spectrum are two Gaussian bands. As is clearly visible the two bands, centred at $2.51 \mathrm{eV}$ (equates roughly to the $2.43 \mathrm{eV}$ band reported elsewhere, discrepancy may be due to interference from electrical contacts) and $2.75 \mathrm{eV}$, show a decrease in intensity as the temperature is increased. A plot of this decrease in intensity is shown in figure 4. Inspection shows that there is a linear decrease in oscillation strength of each electronic transition with temperature. This temperature dependence can largely be ascribed to a decreased intermolecular electronic interaction due to the increased kinetic energy of the spinning spherical molecules. 


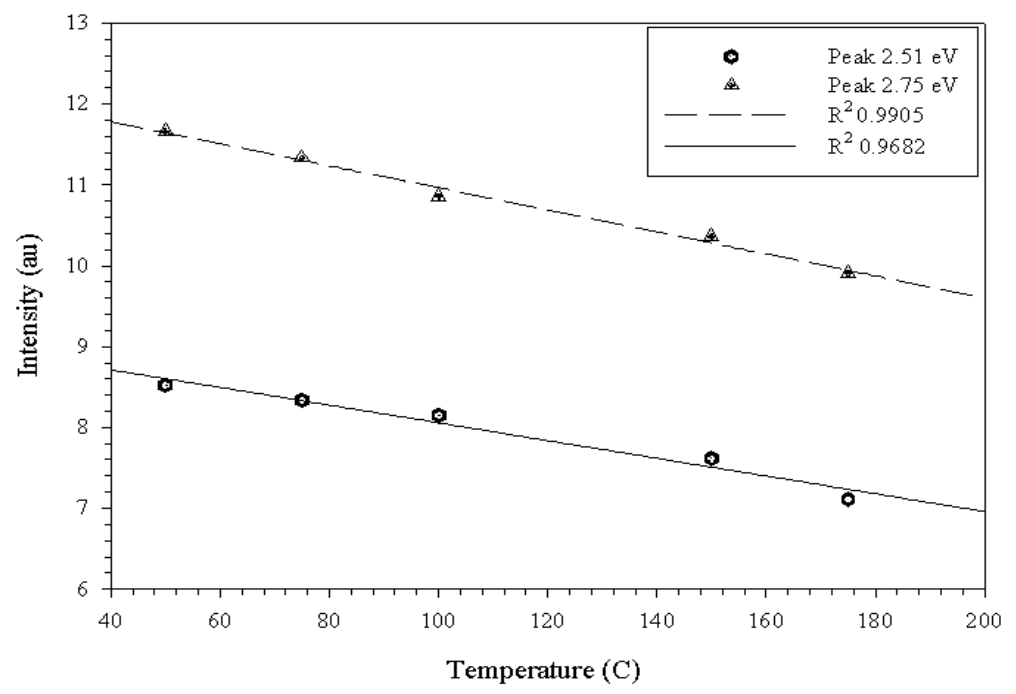

Figure 4 Shows the plot of absorption intensity for two gaussian bands centred at $2.51 \mathrm{eV}$ and $2.75 \mathrm{eV}$ as a function of temperature $\left({ }^{\circ} \mathrm{C}\right)$.

However, it should be noted, that on return to $35^{\circ} \mathrm{C}$, the original spectrum is not recovered. This irreversibility may be ascribed to an annealing process. Once it was confirmed that the samples underwent an annealing process an electroabsorption spectrum of a pristine $\mathrm{C}_{60}$ thin film was obtained. Since the initial aim of this study was to examine the changes in the CT transitions at $2.43 \mathrm{eV}$ and $\sim 2.75 \mathrm{eV}$, the electroabsorption measurements concentrated upon this wavelength region.

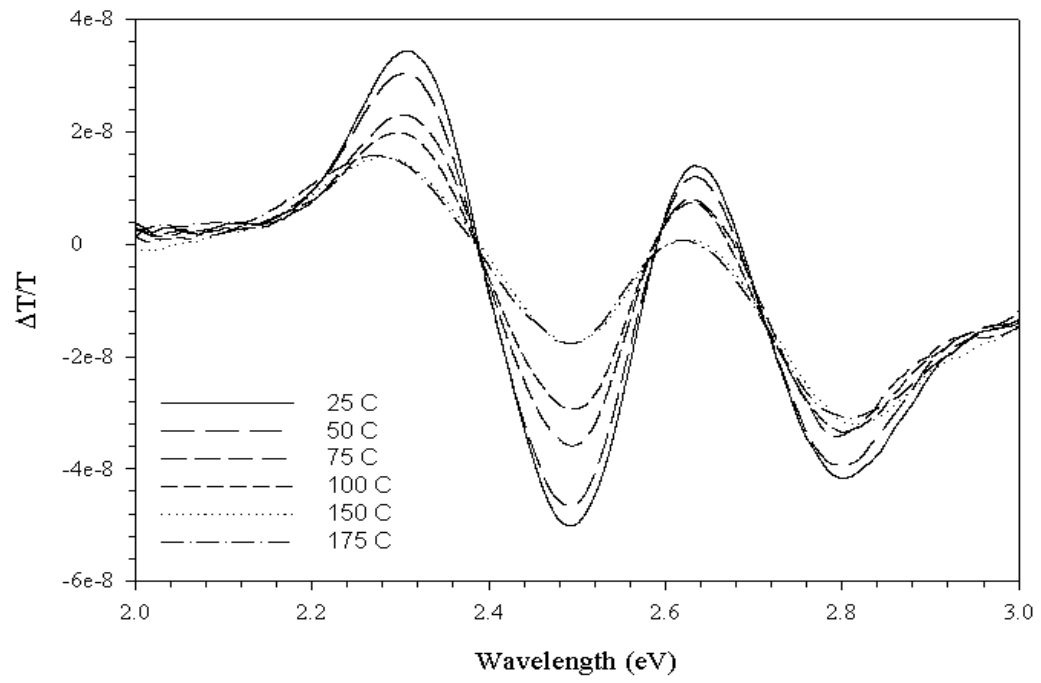

Figure 5 Shows the recorded electroabsorption spectra of a $\mathrm{C}_{60}$ film at various temperatures. The film thickness was $240 \mathrm{~nm}$ as measured using a Dektak 3 surface profiler. The electrical field strength was $500 \mathrm{kV} / \mathrm{cm}$.

Figure 5 shows the recorded electroabsorption spectrum of a $\mathrm{C}_{60}$ film at various temperatures. To maintain consistency, the EA spectra were recorded at the same temperatures as the absorption measurements. Similar to the absorption data, the electroabsorption spectra display a decrease in strength with increasing temperature. Also noticeable is a shifting or broadening of the features at $2.30 \mathrm{eV}$ and $2.62 \mathrm{eV}$. To investigate whether these changes are temperature dependent in nature or due to the annealing process, the film was cooled to room temperature and the electroabsorption spectrum was rerun, as shown in figure 6. 


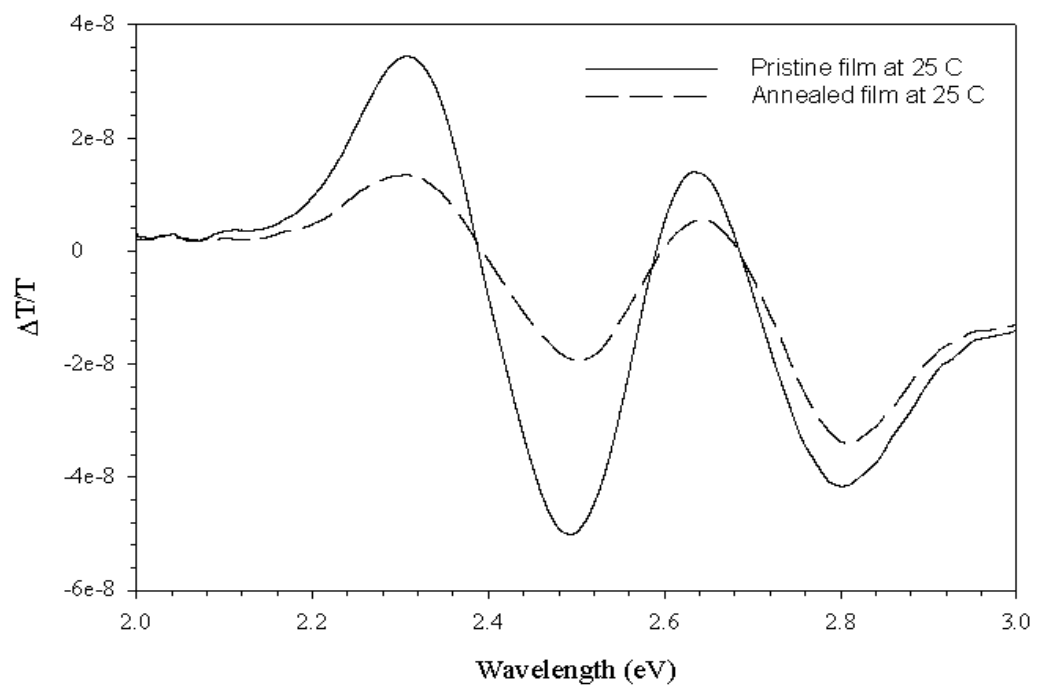

Figure 6 Shows the electroabsorption spectrum of a $\mathrm{C}_{60}$ film prior to and after annealing at $175^{\circ} \mathrm{C}$. Visibly evident is the clear reduction in the feature at $2.43 \mathrm{eV}$, which is derived from a series of CT states created by transferring an electron from the HOMO of one molecule located at the $(0,0,0)$ position the LUMO of its nearest neighbour in the $(1 / 2,1 / 2,0)$ position

Figure 6 shows the EA spectrum for a pristine $\mathrm{C}_{60}$ film, (solid line), at $25^{\circ} \mathrm{C}$. A fit of the spectrum reveals a number of modulations with second order contributions at $2.43 \mathrm{eV}, 2.75 \mathrm{eV}$ and $2.94 \mathrm{eV}$. For the purposes of this work we will concentrate only on the modes located at $2.43 \mathrm{eV}$ and $2.75 \mathrm{eV}$. The positioning of these modes and relative intensities are consistent with those reported in literature for the EA of $\mathrm{C}_{60}$. The strongest feature evident in figure 6 is the mode positioned at approximately $\sim 2.43 \mathrm{eV}$. Previously this mode has been attributed to a Frenkel state due to its resemblance to a first derivative line shape [17]. However subsequent work perform by Tsubo and others attributed this feature to a CT state [18,13] with confirmatory data being provided by Pac et.al. [19]. As figure 6 shows there is a clear reduction in the feature at $\sim 2.43 \mathrm{eV}$, which is derived from a series of CT states created by transferring an electron from the HOMO of one molecule located at the $(0,0,0)$ position to the LUMO of its nearest neighbour in the $(1 / 2,1 / 2,0)$ position of an FCC lattice.

The next most prominent mode is located at $\sim 2.75 \mathrm{eV}$. This mode has been described by Pac. et al as arising from the splitting of the $(1,1,0)$ CT manifold by the off-diagonal charge transfer interactions [19]. There are numerous other CT interactions associated with EA spectrum of $\mathrm{C}_{60}$, and detailed discussions can be found in the various cited works. However in essence the electroabsorption spectrum of $\mathrm{C}_{60}$ can be thought of as a combination of various off diagonal CT interactions coupled with nearby inactive Frenkel states and intensity borrowing from high energy allowed Frenkel excitons. [19]

In order to determine the reliability of the data it was necessary to fit the data with overlapping second derivatives of Gaussian functions. As described elsewhere, [13], the terms associated with the first derivative contributions of the optical absorption represent the intramolecular transitions, and the second derivative contributions represent intermolecular charge transfer transitions. Thus it is possible to determine the degree of contribution each makes to an individual electronic transition using the following model,

$$
d \Delta \alpha=1 / 2 \Delta p F^{2}(d \delta \alpha / \delta E)+1 / 6 \mu^{2} F^{2}\left(d \delta^{2} \alpha / \delta^{2} E\right) \quad \text { Eqn } 1 .
$$

Where $\Delta p, \mu, \alpha, d, F$ and $\mathrm{E}$ are the change in average polarizability tensor, the electric dipole moment, the absorption coefficient, the film thickness, the strength of the electric field and the energy respectively. The $\Delta p$ and $\mu$ are estimated by band resolution of the absorption spectrum into a sum of Gaussian functions and subsequently, fitting the electroabsorption spectrum with a weighted sum of first and second derivatives of these functions. 


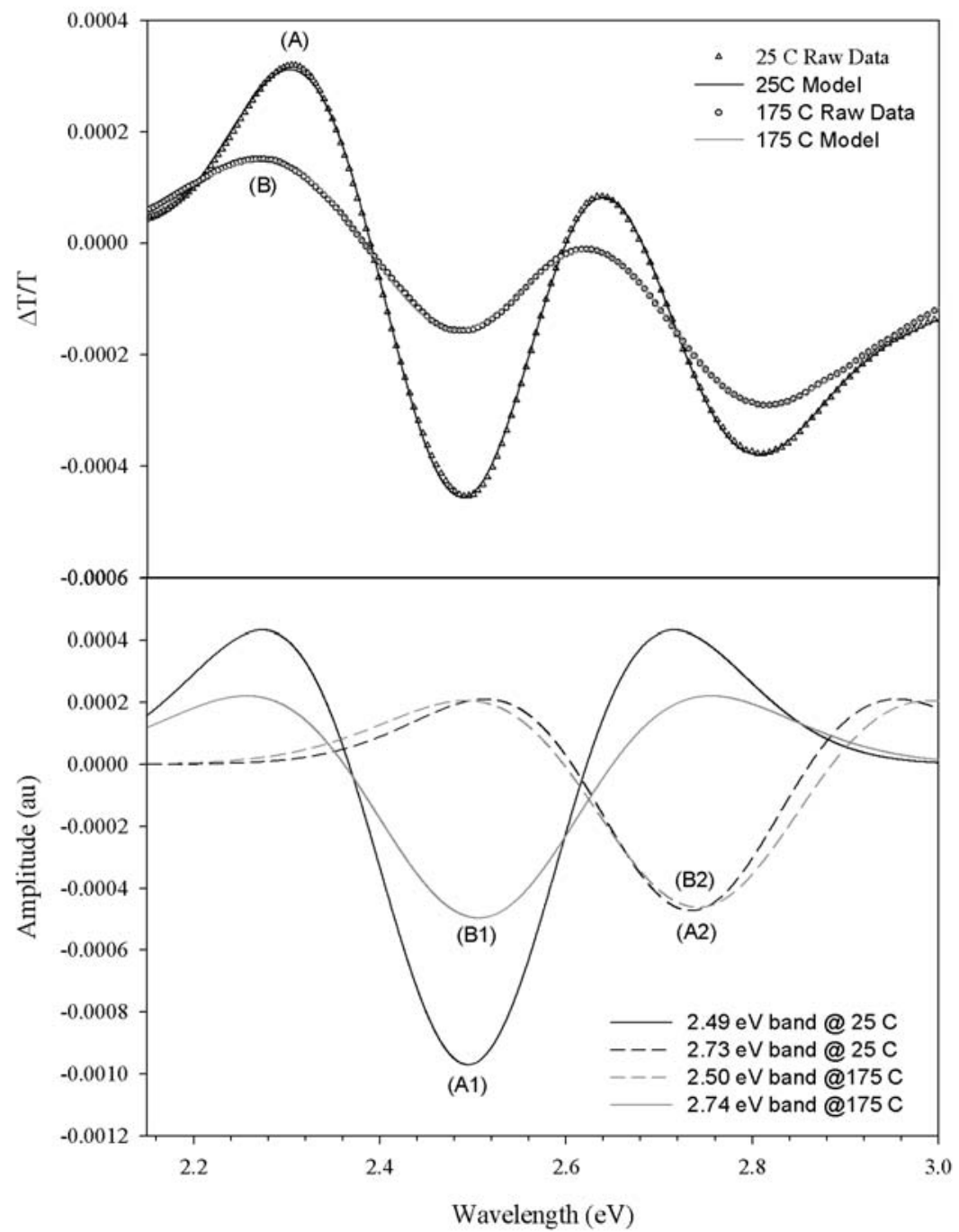

Figure 7 The top graph shows the electroabsorption spectrum and associated model of a $\mathrm{C}_{60}$ film at $25^{\circ} \mathrm{C}$ (curve $\mathrm{A}$ ) and at $175^{\circ} \mathrm{C}$ (curve B). The bottom graph shows the second derivative Gaussian bands as associated with each CT transition. As shown the feature at $\sim 2.7 \mathrm{eV}$ decreases slightly and also displays a small up-shift and broadening of the band. However the feature at $\sim 2.5 \mathrm{eV}$ shows a massive reduction in overall peak intensity.

The top graph in figure 7 shows the fitted electroabsorption spectra for a $\mathrm{C}_{60}$ film at $25^{\circ} \mathrm{C}$ and at $175{ }^{\circ} \mathrm{C}$. The raw data is indicated by the individual symbols. Inspection of the data shows that the fitted model is a close reproduction of the true values. A number of second derivative components, similar to those described by Kazaoui et al [13] are utilised in order to reproduce a good approximation of the raw data. As previously discussed the features at $\sim 2.5 \mathrm{eV}$ and $\sim 2.7 \mathrm{eV}$ are of primary concern and the individual contributions of each second derivative band are show in the bottom graph. Upon inspection, there is a drastic reduction in the feature at $\sim 2.5 \mathrm{eV}$. The feature is associated with a series of CT states created by transferring an electron from the HOMO of one molecule located at the $(0,0,0)$ position the LUMO of its nearest neighbour in the $(1 / 2,1 / 2,0)$ position. Thus the indication is that this transition has been altered due to the increase in temperature of the film. Also it is interesting to note that while the feature at $\sim 2.7 \mathrm{eV}$ shows a small reduction in intensity and band broadening, in the raw data, band resolution analysis of the individual contribution from the $\sim 2.7 \mathrm{eV}$ feature does not show any significant difference between the pristine film and the high temperature. In order to determine whether this decrease is a temperature dependence effect or as a result of the annealing process the individual contribution of each feature needs to be calculated using band analysis for a pristine film and annealed film at room temperature. 


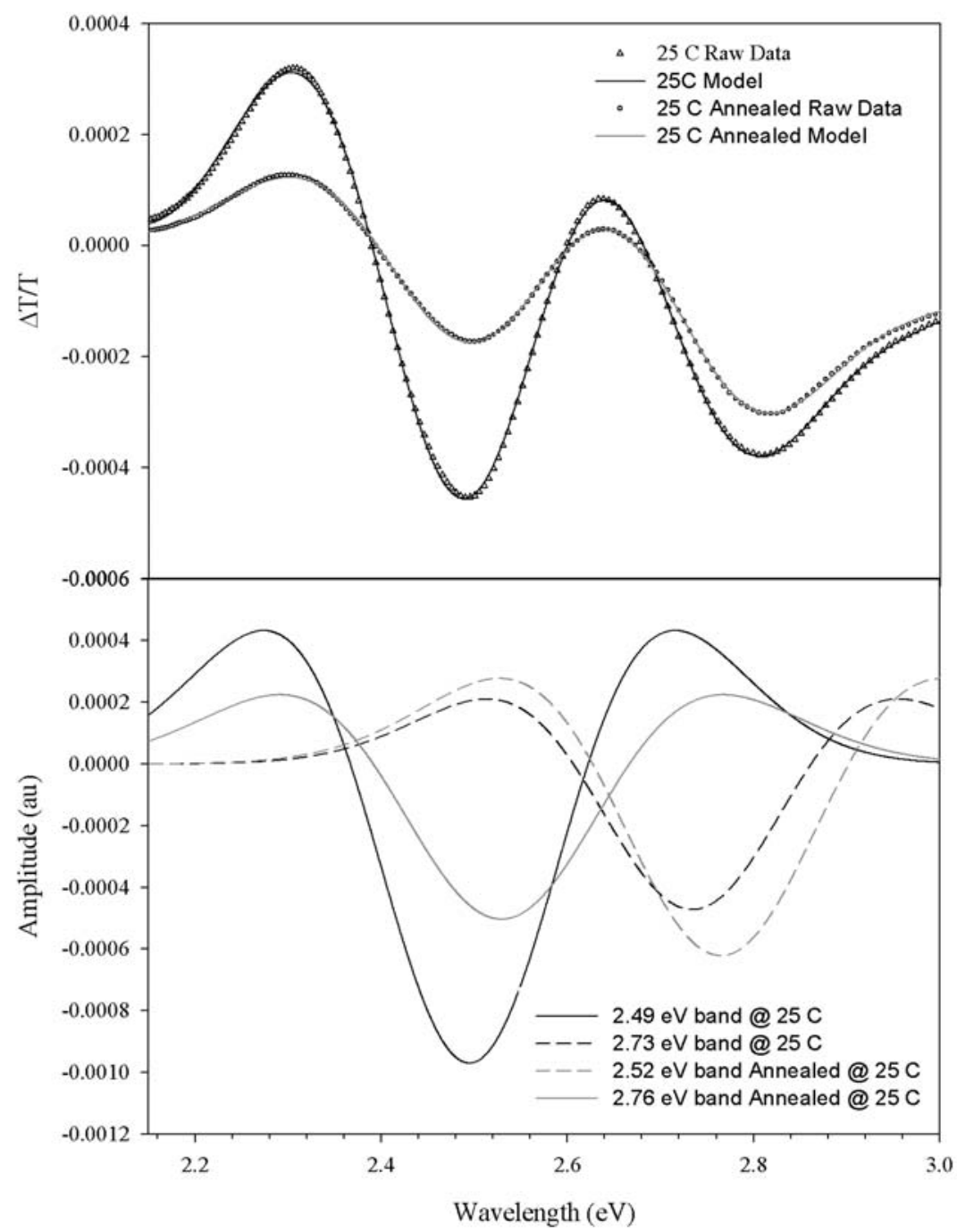

Figure 8 Shows the EA spectrum of a pristine and annealed $\mathrm{C}_{60}$ film. Evident is the clear reduction in the peak intensity at $\sim 2.5 \mathrm{eV}$. There is a also a small reduction in the $\sim 2.7 \mathrm{eV}$ band. Both bands experience a slight up shift in energy, of approximately $0.03 \mathrm{eV}$

The top graph in figure 8 shows the fitted electroabsorption spectra for a pristine $\mathrm{C}_{60}$ film at $25^{\circ} \mathrm{C}$ and also the annealed films at $25^{\circ} \mathrm{C}$. As before the raw data is indicated by the individual symbols and inspection of the data shows that the fitted model is a close reproduction of the true values. As previously discussed the features at $\sim 2.5 \mathrm{eV}$ and $\sim 2.7$ $\mathrm{eV}$ are of primary concern and the individual contributions of each second derivative band are show in the bottom graph. Yet again, as with the high temperature films, the annealed films also exhibit a reduction in the feature at $\sim 2.5 \mathrm{eV}$. Accompanying this reduction is also a slight up shift in energy of the calculated bands. Similarly the feature at $\sim 2.7 \mathrm{eV}$ also experiences a slight up shift in energy, but a smaller reduction in intensity. Thus the suggestion is that the $2.5 \mathrm{eV}$ transition has been altered due to the annealing of the film.

Such a shift may be indicative of a change in the packing density as previously described for the absorption spectrum by introducing changes to the charge transfer species. Indeed annealing processes have been reported to change the $\mathrm{C}_{60}$ lattice from a fcc type arrangement to a hcp type of arrangement, a finding confirmed by $\mathrm{X}$ ray analysis of the films. [6] In addition it has been suggested that the annealing process can improve the electronic properties of $\mathrm{C}_{60}$ films in particular at low annealing temperatures $\left(<300^{\circ} \mathrm{C}\right)[20]$ were it was found that annealed films have a high 
packing density, low surface roughness and high degree of crystallinity and exhibit improved and stable Ohmic contact in comparison to as-deposited $\mathrm{C}_{60}$

With respect to the observed changes in the electroabsorption spectrum it would be expected that any change to the lattice structure or the electronic properties of $\mathrm{C}_{60}$ due to the annealing processes would be readily detected. Since a contraction of the lattice would suggest a small decrease in the distances between adjacent molecules and hence the electron-hole separation of the $(1,0,0)$ lattice coordinate which is reported in literature to be $14.15 \AA$ [13] for pristine $\mathrm{C}_{60}$. As a result of this decrease the $\Delta \mu$ associated. with this CT manifold would also be expected to experience a small decrease.

The presented data would seem to back the assumption up, since $\mathrm{X}$ ray crystallographic data of pristine and annealed films show changes in the packing order as the lattice structure is change from one of an fcc type arrangement to that of a hcp lattice structure. [6] Indeed the data reported by Akselrod et al assign a fcc unit cell for a pristine film with an $a$ value of $14.172 \pm 0.003 \AA$. Annealed films were reported to be a mixture of fcc and hcp, with unit cell dimensions of $a=14.0175 \pm 0.004 \AA$ for the fcc arrangement and $a=9.968 \pm 0.025, c=16.335 \pm 0.003 \AA$ for the hcp arrangement. It was thus the conclusion of Akselrod et al that annealing thin films of $\mathrm{C}_{60}$ not only improved the crystallinity of the films but promoted the formation of a hexagonal phase of $\mathrm{C}_{60}$.

Further analysis is thus under way to fully elucidate, optimise and model the electroabsorption spectrum of annealed $\mathrm{C}_{60}$ films.

\section{CONCLUSION}

The presented work shows that changes in the packing density of fullerene $\mathrm{C}_{60}$ thin films can be monitored using electronic spectroscopy and Electroabsorption (EA)spectroscopy. Characteristic Charge Transfer states have identified at $\sim 2.5 \mathrm{eV}$ and $\sim 2.7 \mathrm{eV}$ and dipole moments are calculated. Thermally annealing of $\mathrm{C}_{60}$ films display both some temperature dependence and partially irreversible effects of these bands, indicating the occurrence of an annealing process. EA shows CT states associated with the transferring an electron from the HOMO of one molecule located at the $(0,0,0)$ position the LUMO of its nearest neighbour in the $(1 / 2,1 / 2,0)$ position has changed position and also experiences a reduction in contribution to the Ea spectrum. Confirmation of this structural change due to the annealing process is provided by previously reported X-Ray crystallographic work.

\section{ACKNOWLEDGEMENTS}

Focas was funded under the National Development Plan 2000-2006 with assistance from the European Regional Development Fund.

\section{REFERENCES}

1. H.W. Kroto, J.R. Heath, S.C. O’Brien, R.F. Curl and R.E. Smalley, Nature 318, 162 (1985)

2. K. Pichler, S. Graham, O.M. Gelsen R.H Friend, W.J. Romanow, J.P. Mc Cauley. N. Coustel, J.E. Fischer, A. B. Smith, J. Phys. Condens. Matter, 3 (1991), 9259

3. P. Petelenz, M. Slawik, B. Pac, Synth Met. 64 (1994), 335

4. K, Kamarás, A. Breitschwerdt, S. Pekker, K. Fodor-Csorba, G. Faigel, M. Tegze. App. Phys. A 56, 231, (1993)

5. L. Akselrod PhD dissertation University of Dublin, Trinity College. (1993).

6. L. Akselrod, H. J Byrne, T. E. Sutto, S. Roth, Chem. Phys. Lett. 233 (1995) 436

7. M. Kaiser, W.K. Maser, H.J. Byrne, A. Mittelbach and S. Roth. Solid State Commun., 87, (1993), 281

8. J. R. Lawrence, G. Chambers, D. Fenton, K Henderson, A.B. Dalton, H.J. Byrne, Synth. Met. 121 (2001), 1145.

9. $\quad$ G.U. Bublitz, S.G. Boxer, Annu, Rev. Phys. Chem., 48 (1997) 213

10. S. Leach, M. Vervloet, A. Desprès, E. Bréheret, J.P. Hare, T. J. Dennis, H.W. Kroto, R. Taylor, and D.R.M. Walton, Chem. Phys., 160, 451, (1992).

11. S. Leach in "Physics and Chemistry of Fullerenes",K.Prassides (ed.) NATO ASI Series, Kuluwer Academic Publishers, 117, (1994), 443. 
12. C. Bulliard, M. Allan and S. Leach, Chem. Phys. Lett., 434, (1993), 209

13. S. Kazaoui, N.Minami, Y. Tanabe, H.J. Byrne, A. Eilmes, P. Petelenz, Phys Rev B. 58, vol 12, (1998), 7689

14. M. Kaiser, J. Reichenbach, H.J. Byrne, J. Anders, W. Maser, S. Roth, A. Zahab and P. Bernier, Solid State Commun., 61, (1992), 81

15. H.J. Byrne, in "Physics and Chemistry of Fullerenes and Derivatives", H. Kuzmany, J. Fink, M. Mehring and S. Roth eds., World Scientific Singapore, (1995), 183

16. X. Wu and S.E. Ulloa, Phys. Rev. B, 7825, (1994), 49.

17. S.Jeglinski, Z.V. Vardeny, D. Moses, V.I. Srdanov, F. Wudl, Synth. Met. 49-50(1992) 557

18. T.Tsubo, K. Nasu, Solid State Commun, 91(1994) 907

19. B.Pac, P. Petelenz, M. Slawik, R.W. Munn, J. Chem. Phys., 109,18 (1998)7932.

20. A. H. Jayatissa, T. Gupta, A. D. Pandya, Carbon, 42, (2004), 1143.

*garrett.farrell@dit.ie 